\title{
Discordant molecular subtype classification in the basal-squamous subtype of bladder tumors and matched lymph-node metastases
}

\author{
Gottfrid Sjödahl $\mathbb{1}^{1,2} \cdot$ Pontus Eriksson ${ }^{3} \cdot$ Kristina Lövgren $^{3} \cdot$ Nour-Al-Dain Marzouka $^{3} \cdot$ Carina Bernardo $^{3}$. \\ Iver Nordentoft ${ }^{4} \cdot$ Lars Dyrskjøt $^{4} \cdot$ Fredrik Liedberg $^{1,2} \cdot$ Mattias Höglund $^{3}$
}

Received: 30 January 2018 / Revised: 25 May 2018 / Accepted: 25 May 2018 / Published online: 2 July 2018

(c) United States \& Canadian Academy of Pathology 2018

\begin{abstract}
Molecular subtypes of muscle-invasive bladder tumors have emerged as a promising research tool with potential to stratify patients for neoadjuvant treatment. Prior to radical cystectomy, the utility of molecular classification and biomarkers depend on concordance between tissue from transurethrally resected specimens and disseminated disease. We assess the concordance of molecular subtypes and a large number of potential biomarkers in 67 pairs of muscle-invasive bladder tumors and synchronous lymph-node metastases. Tissue cores were stained for 29 immunohistochemistry markers and immunohistochemistry-based molecular subtype classification was performed. Molecular subtype was determined by mRNA profiling for 57 bladder tumors and 28 matched lymph-node metastases. Full section immunohistochemistry was performed to assess intra-tumor subtype heterogeneity in discordant cases, and exome sequencing was performed for 20 sample pairs. Discordant subtype classification between the bladder tumor and lymph-node metastasis was generally rare (12/67, 18\%), but most $(7 / 12,58 \%)$ involved the Basal/Squamous-like subtype. Discordant Basal/Squamous-like tumors showed either Urothelial-like or Genomically Unstable, luminal-like phenotype in the lymph-node metastasis. Full section immunohistochemistry revealed intra-tumor subtype heterogeneity for six discordant cases including four involving the Basal/Squamous-like subtype. Subtype concordance for non- Basal/Squamous-like tumors was 91\%. RNA-based classification agreed with immunohistochemistry classification but quantitative assessment is necessary to avoid false detection of subtype shifts. Most high confidence cancer mutations were shared between samples $(n=93,78 \%)$, and bladder tumor private mutations ( $n=20,17 \%)$ were more frequent than those private to the lymph-node metastasis $(n=7,6 \%)$. We conclude that bladder tumors and lymph-node metastases have overall similar molecular subtype, biomarker expression, and cancer mutations. The main exception was tumors of the Basal/Squamous-like subtype where most cases showed discordant classification, some with evidence of intra-tumor heterogeneity. The data are of relevance for neoadjuvant treatment stratification and raises questions on the dynamics of molecular subtypes during bladder cancer progression.
\end{abstract}

Electronic supplementary material The online version of this article (https://doi.org/10.1038/s41379-018-0096-5) contains supplementary material, which is available to authorized users.

$\triangle$ Gottfrid Sjödahl

gottfrid.sjodahl@med.lu.se

1 Division of Urological Research, Department of Translational Medicine, Lund University, Lund, Sweden

2 Department of Urology, Skåne University Hospital, Malmö, Sweden

3 Division of Oncology and Pathology, Department of Clinical Sciences, Lund University, Lund, Sweden

4 Department of Molecular Medicine (MOMA), Department of Clinical Medicine, Aarhus University Hospital and Aarhus University, Aarhus, Denmark
Muscle-invasive bladder cancer is a lethal disease with only half of the patients surviving despite radical cystectomy [1]. Patients with node-positive disease have further decreased survival estimates. Neoadjuvant chemotherapy gives a modest but significantly improved outcome compared to cystectomy alone [2]. Recently, immune-checkpoint inhibitors have emerged as a treatment option in the metastatic setting for unfit patients and in the second-line setting after chemotherapy [3-5]. However, an increased survival for neoadjuvant chemotherapy of about 5\% [6] and response rates of checkpoint inhibitors between $15 \%$ and $24 \%$ suggest that most patients do not benefit from either treatment. So far, efforts to predict therapeutic response have studied single biomarkers [7, 8], molecular subtypes [9, 10], or mutations [11-13]. Studies investigating predictive 
biomarkers have assumed primary tumors to be representative of the disseminated disease. Indeed, comparisons between primary tumors and matched lymph-node metastases show good concordance for ploidy and clonality $[14,15]$. Studies investigating expression of single genes/ proteins, e.g. ERBB2 [16-18], EGFR [19], FGFR3 [20, 21], CDH1 [22], and CCND1 [23] show concordance rates ranging from $69 \%$ to $100 \%$, indicating that lymphnode metastases are stable with regard to genetic origin as well as to single biomarkers.

Two main methods have been used to define molecular subtypes of bladder cancer-global mRNA-based classification [9, 24-26] and in situ immunohistochemistry-based tumor-cell classification [27, 28]. Distinct systematic discrepancies between the two methods have however been described, mainly caused by variation in the amount of sampled non-tumor cells [28]. With the exception of subtypes driven by strong immune/stromal content, we have identified five main molecular subtypes using both mRNA- or immunohistochemistry-based classification; the Luminal-like subtypes Urothelial-like and Genomically Unstable that both express a urothelial-differentiation signature. Urothelial-like tumors also express FGFR3 and are enriched for papillary histology, whereas Genomically Unstable tumors have lost cell-layer stratification, cell-cell adhesion, and show molecular profiles similar to carcinoma in situ. The Basal/Squamous-like subtype shows overexpression of basal urothelial markers, i.e. KRT5 and CDH3 (P-Cadherin) but also a squamous keratinization signature not expressed in normal urothelium. Additionally, two minor urothelial-differentiation negative bladder cancer subtypes are also included in the Lund taxonomy, Mesenchymal-like and Small-cell/neuroendocrine-like, enriched for, but not exclusively comprising, histological variant tumors [26, 28].

Studies comparing molecular subtypes between primary tumors and metastases have been performed for other tumor types, but not for bladder cancer. In breast cancer, molecular subtype concordance of brain metastases was 53-85\% with increased HER2-enriched subtypes in metastases $[29,30]$.

Here we investigate to what extent molecular subtypes differ between a diagnostic transurethral resection sample and disseminated disease. We sampled all available lymphnode metastases from the cystectomy-lymphadenectomy specimens of a large consecutive cohort of 425 patients undergoing radical cystectomy for bladder cancer in southern Sweden. This allowed us to perform molecular subtype classification by global mRNA profiling and/or immunohistochemistry for 67 primary tumor transurethral resection samples and their matched synchronous lymphnode metastases. For 20 of the 67 patients, tissue was sufficient for exome sequencing, which was used to identify known cancer mutations that were either private or shared between primary tumors and matched lymph-node metastases.

\section{Materials and methods}

\section{Patients and tissue blocks}

Formalin-fixed paraffin embedded muscle-invasive primary tumor and lymph-node metastases tissues were obtained from transurethral resection of the bladder and cystectomylymphadenectomy specimens, respectively, from a population-based series of 425 patients undergoing radical cystectomy between 2006 and 2011 [28]. Node-positive cases were included if primary and lymph-node metastases tumor tissues could be obtained $(n=67)$. Clinical, pathological, and outcome patient data are summarized in Table 1. Surgery was performed as an extended lymphadenectomy up to the ureteric crossing or aortic bifurcation. Eight patients received neoadjuvant chemotherapy with dose-dense Metotrexate, Vinblastine, Adriamycin, Cisplatin neoadjuvant combination chemotherapy. Three patients received induction chemotherapy, defined as definitive treatment in clinically node-positive disease: one patient with small-cell carcinoma received induction chemotherapy with cisplatin and etoposide and two received the cisplatinbased combination. The regional ethical review board in Lund approved the study (EPN Lund2012/22).

\section{Immunohistochemistry}

Tissue microarrays containing two $1.0 \mathrm{~mm}$ tissue cores per sample were constructed and a panel of 29 primary antibodies was applied. The two cores were placed in the transurethral resection specimen blocks without consideration of spatial separation. The two cores therefore originated from different tissue pieces within the resected material for the majority of cases, but sometimes, particularly when the total tumor cell area is small, they originated from the same tissue piece. The antibody panel and staining procedures used for this cohort have been described in detail [28]. Stained slides were scanned (AxioScan Z1, Zeiss) and evaluated using the digital pathology platform PathXL. Markers were evaluated on an intensity scale (0-3), a percentage scale $(0-9,10 \%$-bins), or both. The score for each tumor was calculated as the mean values of the two cores. Immunohistochemistry-based subtype classification into "Urothelial-like", "Genomically Unstable", "Mesenchymallike", "Basal/Squamous-like", and "Small-cell/Neuroendocrine-like" was performed using four-marker subtype panels as described [28]. To assess intra-tumor heterogeneity, immunohistochemistry for selected markers was applied, 
Table 1 Clinical and pathological characteristics of the 67 patients included in the study

\begin{tabular}{lll}
\hline Clinical, pathological, and outcome data & \\
\hline \multicolumn{4}{l}{$\begin{array}{l}\text { Discordant } \\
\text { subtype }(n=12)\end{array}$} & $\begin{array}{l}\text { Concordant } \\
\text { subtype }(n=55)\end{array}$ & Total $(n=67)$ \\
\hline
\end{tabular}

\begin{tabular}{|c|c|c|c|}
\hline \multicolumn{4}{|l|}{ Age, years } \\
\hline Mean (SD) & $70.8(5.4)$ & $68.9(8.3)$ & $69.3(7.9)$ \\
\hline $\begin{array}{l}\text { Median } \\
\text { (range) }\end{array}$ & $\begin{array}{l}72.6 \\
(61.9-77.3)\end{array}$ & $68.7(50.6-85.3)$ & $\begin{array}{l}69.9 \\
(50.6-85.3)\end{array}$ \\
\hline \multicolumn{4}{|l|}{ Gender, $n(\%)$} \\
\hline Female & $1(8.3)$ & $10(18.1)$ & $11(16.4)$ \\
\hline Male & $11(91.7)$ & $45(81.8)$ & $56(83.6)$ \\
\hline \multicolumn{4}{|c|}{ Clinical stage, $n(\%)$} \\
\hline cis & $1(8.3)$ & 0 & $1(1.5)$ \\
\hline $\mathrm{T} 1$ & $1(8.3)$ & $4(7.3)$ & $5(7.5)$ \\
\hline $\mathrm{T} 2$ & $4(33.3)$ & $24(43.6)$ & $28(41.8)$ \\
\hline $\mathrm{T} 3$ & $4(33.3)$ & $19(34.5)$ & $23(34.3)$ \\
\hline $\mathrm{T} 4$ & $2(16.7)$ & $8(14.5)$ & $10(14.9)$ \\
\hline \multicolumn{4}{|c|}{ Pathological stage, $n(\%)$} \\
\hline pT0 & $1(8.3)$ & 0 & $1(1.5)$ \\
\hline pTis & 0 & $1(1.8)$ & $1(1.5)$ \\
\hline pT1 & 0 & $2(3.6)$ & $2(3.0)$ \\
\hline pT2a & 0 & $4(7.3)$ & $4(6.0)$ \\
\hline pT2b & 0 & $7(12.7)$ & $7(10.4)$ \\
\hline pT3a & $1(8.3)$ & $11(20.0)$ & $12(17.9)$ \\
\hline pT3b & $7(58.3)$ & $12(21.8)$ & $19(28.4)$ \\
\hline pT4a & $1(8.3)$ & $16(29.1)$ & $17(25.4)$ \\
\hline pT4b & $2(16.7)$ & $2(3.6)$ & $4(6.0)$ \\
\hline \multicolumn{4}{|c|}{ Clinical node status, $n(\%)$} \\
\hline No & $11(91.7)$ & $48(87.3)$ & $59(88.1)$ \\
\hline N1 & 0 & $3(5.5)$ & $3(4.5)$ \\
\hline $\mathrm{N} 2$ & 0 & $2(3.6)$ & $2(3.0)$ \\
\hline M1 & $1(8.3)$ & $2(3.6)$ & $3(4.5)$ \\
\hline \multicolumn{4}{|c|}{ Pathological node status, $n(\%)$} \\
\hline $\mathrm{pN} 1$ & $1(8.3)$ & $17(30.9)$ & $18(26.9)$ \\
\hline $\mathrm{pN} 2$ & $10(83.3)$ & $34(61.8)$ & $44(65.7)$ \\
\hline $\mathrm{pN} 3$ & $1(8.3)$ & $4(7.3)$ & $5(7.5)$ \\
\hline \multicolumn{4}{|c|}{ Neoadjuvant chemotherapy, $n(\%)$} \\
\hline None received & $11(91.7)$ & $48(87.3)$ & $59(88.1)$ \\
\hline $\begin{array}{l}\text { Dose-dense } \\
\text { MVAC }\end{array}$ & $1(8.3)$ & $4(7.3)$ & $5(7.5)$ \\
\hline $\begin{array}{l}\text { Cisplatin } \\
\text {-based } \\
\text { induction } \\
\text { chemo }\end{array}$ & 0 & $3(5.5)$ & $3(4.5)$ \\
\hline \multicolumn{4}{|l|}{ Survival, $n(\%)$} \\
\hline Alive & $5(41.7)$ & $5(9.1)$ & $10(14.9)$ \\
\hline $\begin{array}{l}\text { Death, bladder } \\
\text { cancer }\end{array}$ & $6(50.0)$ & $46(83.6)$ & $52(77.6)$ \\
\hline $\begin{array}{l}\text { Death, other } \\
\text { cause }\end{array}$ & $1(8.3)$ & $4(7.3)$ & $5(7.5)$ \\
\hline
\end{tabular}

under the same conditions as with tissue microarray staining, to the full section block from the transurethral resection or from lymph-node metastases. Cases were considered to show subtype heterogeneity if a continuous area of tumor cells with the minimum size of a $1 \mathrm{~mm}$ tissue core showed opposite staining compared to the originally sampled tissue cores. According to this definition, single cells or small clusters of cells with opposite staining did not constitute subtype heterogeneity.

\section{DNA and RNA extraction}

Tissue blocks were sectioned and stained with hematoxylin and eosin $(H \& E)$. $H \& E$ slides were then used to guide macro-dissection by etching around a high tumor-content area of the paraffin block with an awl. Depending on the size of the macro-dissected area, 4-10 $10 \mu \mathrm{m}$ sections were taken, and paraffin flakes were transferred to Eppendorf tubes. Deparaffinization using serial incubations of xylene and ethanol, overnight lysis and proteinase $\mathrm{K}$ digestion, and RNA/DNA extraction (HighPure, Roche) were then performed according to the manufacturer's instructions. lymph-node metastases samples with minimal or no tumor tissue left after tissue microarray construction were excluded from RNA or DNA analyses.

\section{Gene expression profiling and subtype classification}

Data from all bladder tumors used here are publicly available as part of a larger cohort of 307 muscle-invasive tumors in Gene Expression Omnibus under GSE83586. In addition, 28 lymph-node metastasis samples not previously published are now made accessible through GSE101723. Assignment of gene expression subtypes was performed as described in the original study with the additional lymphnode metastasis samples added to the data set. First, all samples were subjected to stepwise two-way splits by consensus clustering on $50 \%$ variance filtered data. This was performed after RMA normalization and median centering together with the full GSE83586 data set (total $n 307+28=335)$. Each sample formed part of one out of seven clusters identified in the original study ("Urotheliallike A", "Genomically Unstable", "Infiltrated", "Basal/ SCC-like", "Mesenchymal-like","Urothelial-like B", and "Small-cell/neuroendocrine-like"). Classification according to the TCGA into "Luminal Papillary", "Luminal", "Luminal Immune", "Basal/Squamous", and "Neuronal" were kindly provided by Jaegil Kim and colleagues [26].

\section{Exome sequencing}

DNA concentration was calculated using a Qubit 3.0 fluorometer (ThermoFisher). Exome-Seq library construction 
was made using the KAPA HyperPlus Library Kit (Kapa Biosystems by Roche) and NimbleGen SeqCap EZ MedExome (Roche). Genomic DNA was fragmented by a Covaris sonication device (E220evolution) to a fragment size of 200-250 bp (duty cycle: 10\%; peak incident power: 175; cycles per burst: 200; time (seconds): 50 ; temperature $\left({ }^{\circ} \mathrm{C}\right)$ : $6-8$; water level ( $\mathrm{S}$ series): 6; volume ( $\mu \mathrm{L})$ : 52.5) using $10-100 \mathrm{ng}$ input. Two libraries were made for each DNA sample. Libraries were quantified using adaptor specific QPCR (KAPA Library Amplification Kit, Roche), combined in pools of up to eight libraries (500-1000 ng) and captured using the NimbleGen protocol modified to accommodate pooled indexed libraries (SeqCap EZ Library SR User Guide v4.2). The libraries were sequenced (Paired end $2 \times 75 \mathrm{bp}$ ) using the Illumina NextSeq 500 platform. Variants were called using GATK HaplotypeCaller and filtered by removing copy number variants and variants with two different alternate alleles. Variants described in dbSNP were removed, and remaining variants annotated using snpEff v4.3 and the GRCh37.75 database as high, moderate, or low were kept. Out of 28 sample pairs, 4 did not co-cluster based on data from polymorphic loci and were excluded. Another four pairs were excluded due to low coverage for either the primary tumor or the metastasis sample; leaving 20 sample pairs and 8 matching normal DNA samples (negative lymph nodes from the lymphadenectomy specimen) for mutation analysis. Minimum 10 reads coverage and 3 reads supporting the alternate allele were required. For final use of the data, we identified high confidence cancer mutations including variants included either in the COSMIC cancer gene census [31] or among significantly mutated genes in bladder cancer (189 genes, TCGA cohort $n=395$ ). High confidence mutations were also absent in the ExAC database [32], previously reported as somatic in COSMIC, with predicted deleterious effect at protein level as assessed by PolyPhen-2 [33] or with loss of function intolerance annotation in ExAC. By these methods, we identified 120 high confidence mutations occurring in 19/20 patients. Fifty-four of these were absent in matched normals, and only two mutations initially on the high confidence mutation list were removed due to presence in the normal sample, indicating that most mutations in the full list (Supplementary Table 1) are somatic.

\section{Statistical tests}

For pairwise tests, we used the mean value for the cases with two lymph-node metastasis samples. Statistical tests were two-sided with $\alpha=0.05$ considered statistically significant unless specifically indicated. All statistical calculations were performed in $\mathrm{R}$.

\section{Results}

\section{Urothelial-like and Genomically Unstable molecular subtypes are concordant in lymph-node metastases}

Of 67 bladder tumors, 48 were immunohistochemistryclassified either as Urothelial-like or Genomically Unstable subtypes, which are phenotypically distinct entities of the broader luminal-like class of muscle-invasive bladder cancer. The analysis revealed that 46 of the 48 primary tumors unequivocally classified as Urothelial-like or Genomically Unstable showed a concordant immunohistochemistry classification in lymph-node metastases. Of the two discordant cases, one Urothelial-like case showed an atypical-, and one a Basal/Squamous-like subtype in the metastasis (Fig. 1a). Gene expression profiles for 57 of the primary tumors and 28 of the lymph-node metastases were also used to generate mRNA subtype classification by co-clustering in the extended data set (Fig. 1b). Differences between mRNA- and immunohistochemistry classification mainly involved Urotheliallike/Genomically Unstable cases (immunohistochemistry) classified as Infiltrated by mRNA, and Urothelial-like cases (immunohistochemistry) being more specifically subclassified as Urothelial-like $B$ by mRNA. These discrepancies are expected consequences of classification at the tumor-cell versus global mRNA level. Global mRNA classification cannot identify specific subtypes when highly infiltrated, and immunohistochemistry classification does not distinguish Urothelial-like A from Urothelial-like B. In addition to these systematic differences in classification, four Urotheliallike cases remained discrepant between immunohistochemistry and mRNA (two classified as Basal/Squamous-like and two classified as Genomically Unstable). To further compare mRNA and immunohistochemistry classification, we applied the Lund2012 nearest-centroid classifier (Supplementary Figure 1, also showing the 5-group TCGA classification). By quantifying each sample's correlation to five subtype centroids we observed that cases with discrepancy between mRNA and immunohistochemistry classification showed ambiguous mRNA centroid correlations. In those cases, a threshold effect could cause very small changes in centroid correlations to result in a change in subtype classification (exemplified as false positive subtype shifts in Fig. 2). Cases with ambiguous classification due to this threshold effect had a subtype distribution more similar to cases concordant by both methods than to cases discordant by both methods (Fig. 2a, d, g). For each Primary-Metastasis sample pair, radar plots of mRNA centroid correlations and immunohistochemistry-subtype scores were used to visualize the classification quantitatively (mRNA: 1-Pearson- $r$ distance to each of five centroids; immunohistochemistry: 4marker scores on Genomically Unstable-Urothelial-like axis as well as on separate axes for Basal/Squamous-like, 


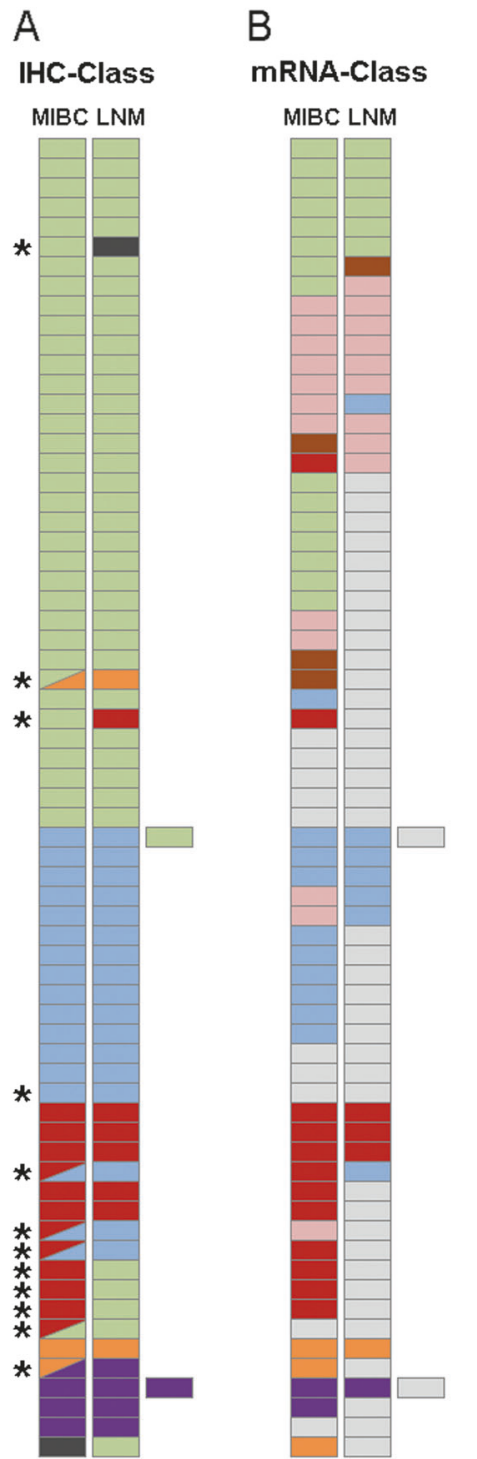

Fig. 1 Bladder tumors and matched lymph-node metastases show high subtype concordance with the exception of tumors of the Basal/ Squamous subtype. Each row shows data for one patient. a Molecular subtype classification using immunohistochemistry. The first column shows immunohistochemistry-subtype of primary tumors, the second column of lymph-node metastases, and for two cases a second metastasis is included in the third column. Cases where both the primary tumors and lymph-node metastases were investigated by full section are marked with a star. Cases with marked subtype

Mesenchymal-like, and Small-cell/neuroendocrine-like). Figures $2 \mathrm{~b}$, e, h exemplify that immunohistochemistry-discordant cases showed large difference in shape of the radar plots both for mRNA- and immunohistochemistry-subtype scores. On the contrary, cases demonstrating the threshold effect were only nominally discordant in mRNA classification, as the differences in radar plots were similar to fully concordant cases. Most importantly, immunohistochemistry of the same cases confirmed that there was no subtype discordance (Fig. 2c, f, i). Exome-seq data for Primary-Metastasis sample heterogeneity in full section are indicated with split boxes colored according to the observed subtypes. b Molecular subtype classification by co-clustering of gene expression data. Each box represents the same sample as shown in a. Gray boxes indicate that gene expression data were not available. $\mathbf{c}$ Each box represents one mutation, color coded according to its status as shared or private. Gene symbols for 1-2 selected shared mutations (left) and for all private mutations (right) are shown. MIBC muscle-invasive bladder cancer, LNM lymph-node metastasis, ITH intra-tumor heterogeneity

pairs was generated for 20 cases with a total of 120 high confidence mutations identified (Supplementary Table 1). For each patient, selected shared mutations and all private mutations are indicated in Fig. 1c. Genomically Unstable cases showed a higher proportion of private mutations than Urothelial-like (Fisher's exact test, $p=0.0015$ ). Taken together, immunohistochemistry classification, mRNA profiling, and mutations all indicate that lymph-node metastases of the Urothelial-like subtype are near identical to the corresponding primary tumors. Similarly, the Genomically Unstable 
A

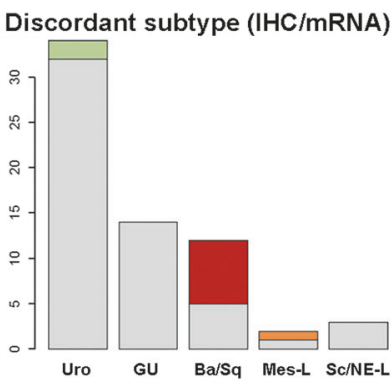

D

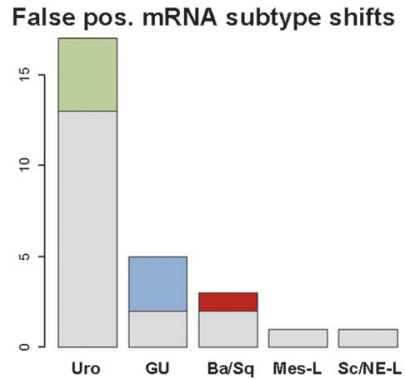

G

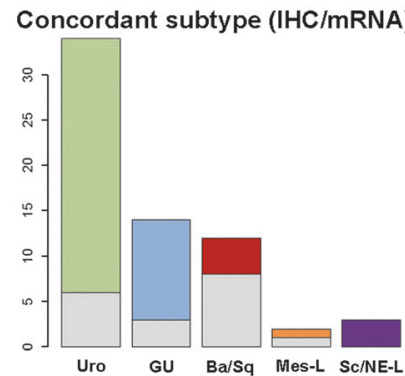

B

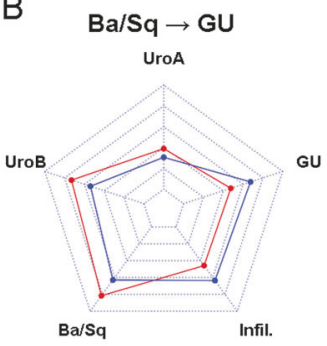

$E$
UroB

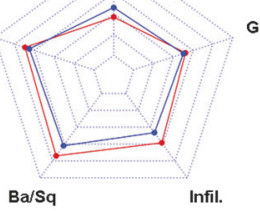

GU

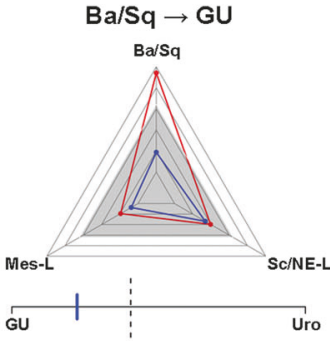

Gu

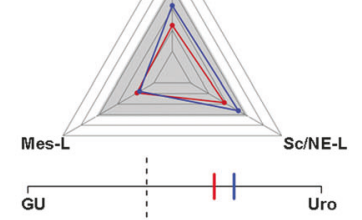

$\mathrm{H}$
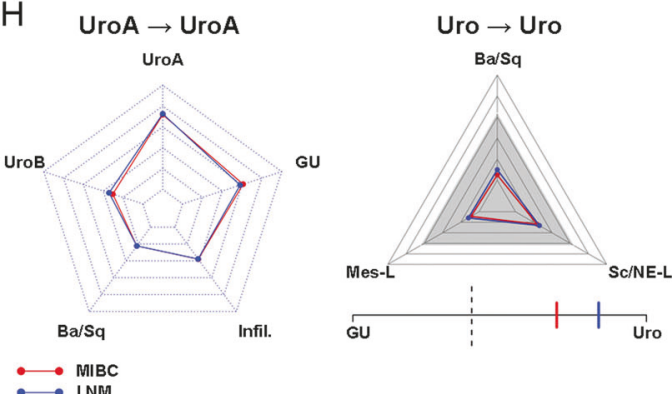

Fig. 2 Threshold effects in subtype classification lead to an overestimated prevalence of subtype shifts. a Barplot showing the proportions of each immunohistochemistry-subtype with discordant subtype classification. b Example of a quantitative analysis of subtype classification for a Basal/Squamous-like to Genomically Unstable discordant case. Red dots/lines show primary tumor values and blue dots/lines metastasis values. The pentagonal radar plot shows the paired samples' correlation to the Lund2012 centroids. The triangular radar plot shows the immunohistochemistry classification scores: Scores on three panels result in classification as Basal/Squamous-like, Small-cell/neuroendocrine-like, or Mesenchymal-like when above cut-off (gray area). When below cut-off on all three axes, the Genomically Unstable-Urothelial-like axis determines subtype (dotted line indicates the cut-off value). c Selected immunostainings

molecular subtype was fully concordant in the corresponding lymph-node metastases, but did show a higher number of private mutations (Fig. 1b).

\section{Basal/Squamous-like bladder tumors frequently show Urothelial-like or Genomically Unstable subtype in lymph-node metastases}

Among the identified 12 primary tumors with Basal/Squamous-like phenotype seven revealed a discordant Urothelial-like or Genomically Unstable subtype
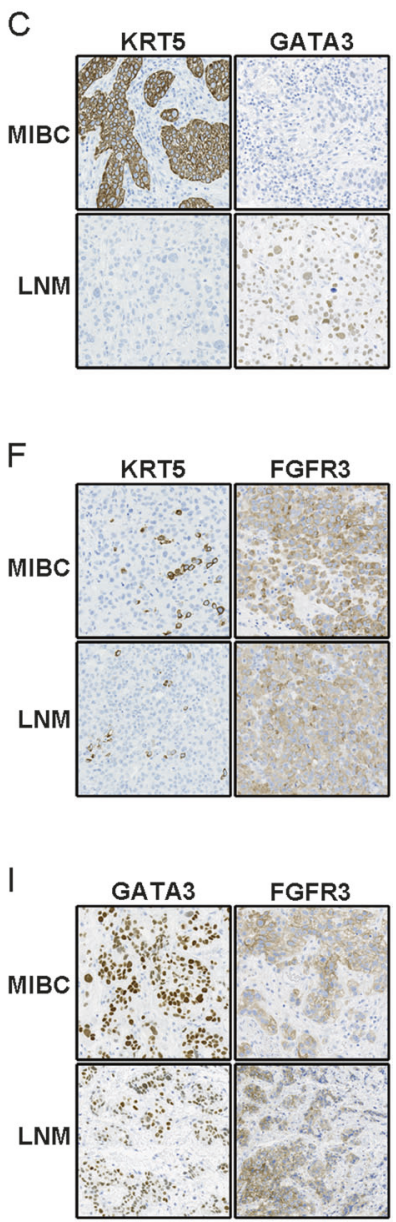

for the same case as in b. d Barplot showing the proportions of each immunohistochemistry-subtype with discordant subtype by centroid-based mRNA classification $(n=28)$, not validated by quantitative analysis or immunostaining. e Example of a quantitative analysis of subtype classification showing a "threshold effect", i.e. a false discordant result. Data are shown as described in b. f Selected immunostainings for the same case as in e, showing no true subtype discordance. $\mathbf{g}$ Barplot showing the proportions of each immunohistochemistry-subtype with fully concordant subtype classification. h Example of a quantitative analysis of subtype classification for a concordant Urothelial-like tumor. i Selected immunostainings for the same case as in $\mathbf{h}$. Tumor images are $300 \times$ $300 \mu \mathrm{m}$. MIBC muscle-invasive bladder cancer, LNM lymph-node metastasis

classification in the lymph-node metastases. RNA- and immunohistochemistry classification was in agreement for Basal/Squamous-like cases, including one case classified as Genomically Unstable in the metastasis sample by both methods (Fig. 1a). This case showed subtype heterogeneity (Basal/Squamous-like and Genomically Unstable) in the primary tumor with only the Genomically Unstable phenotype found in the lymph-node metastasis. However, full section immunohistochemistry for KRT5 and GATA3 revealed that the primary tumor area from which DNA and RNA was extracted contained exclusively 
A
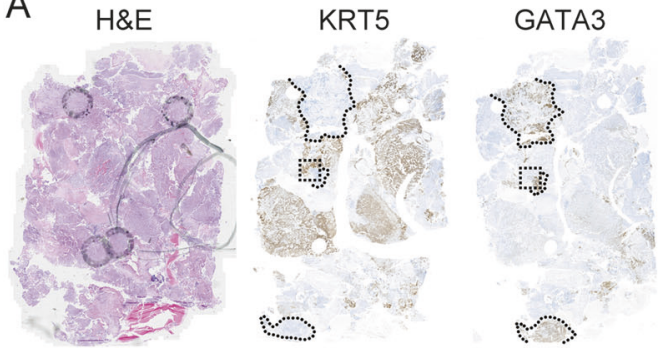

C
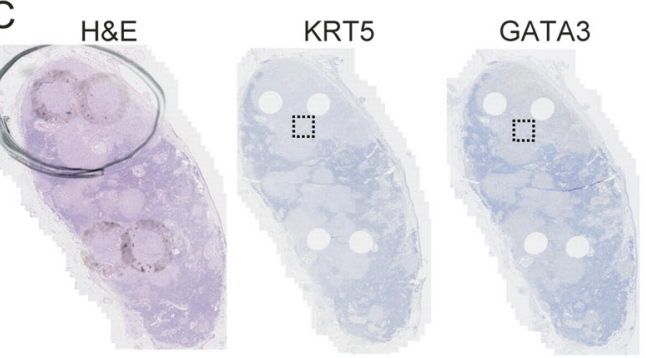
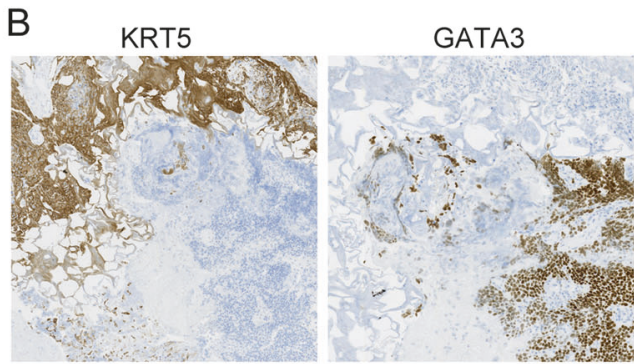

D

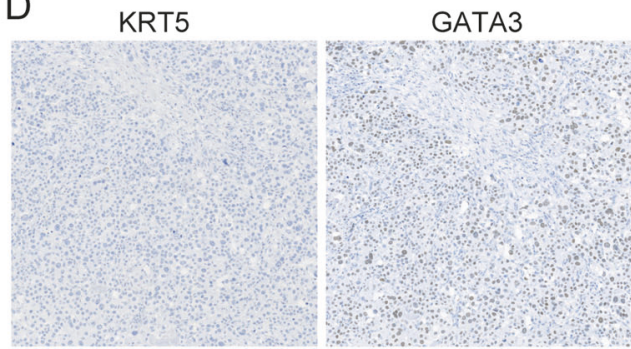

Fig. 3 Full section immunostainings reveal subtype discordance associated with intra-tumor heterogeneity. a Full section (transurethral resection) stains of a Basal/Squamous-like primary tumor and the discordant Genomically Unstable lymph-node metastasis. This case showed molecular subtype heterogeneity in the primary tumor but not in the lymph-node metastasis. On the H\&E stain, the marked areas indicate placement of TMA cores, and macro-dissected areas for DNA/ RNA extraction. Dotted lines indicate border between molecular subtypes. b One millimeter squares from the indicated area in the corresponding full sections shown in higher magnification. The area

Basal/Squamous-like tumor (Fig. 3a). Only one high confidence cancer mutation was identified for this patient: the identical ERBB3 driver mutation (V104L) was present in the Basal/Squamous-like area of the primary tumor and the area of the metastasis classified as Genomically Unstable, at allele frequencies 0.32 and 0.20 , respectively, indicating a clonal relationship.

\section{Subtype intra-tumor heterogeneity was observed in half of the discordant bladder tumors}

We were able to perform full section immunohistochemistry in 12 cases for both the primary tumor and lymph-node metastasis blocks, all except one that showed discordant immunohistochemistry-subtype classification (Fig. 1a). Two to four markers were applied for each case selected based on the results from the initial analysis on tissue microarray cores. In 6 of 12 analyzed cases, full section immunohistochemistry indicated subtype intra-tumor heterogeneity in the primary tumor tissue block (Fig. 3, all cases shown in Supplementary Figure 2) whereas intratumor heterogeneity never was observed in the lymph-node metastases. Primary tumor subtype heterogeneity was observed once between Urothelial-like and Mesenchymallike, in three instances between Basal/Squamous-like covers the border between molecular subtypes. c No heterogeneity was observed in full section stains of the lymph-node metastasis sample. On the H\&E stain, the marked areas indicate placement of TMA cores, and macro-dissected areas for DNA/RNA extraction. d One millimeter squares from the indicated area in the corresponding full sections shown in higher magnification. Presence of nuclear GATA3 staining, albeit weaker than in the primary tumor, and lack of KRT5 staining, confirmed the discordant Genomically Unstable subtype classification compared to the heterogeneous but mainly Basal/Squamous-like primary tumor. H\&E hematoxylin and eosin

and Genomically Unstable, and once each between Basal/Squamous-like and Urothelial-like, and between Mesenchymal-like and Small-cell/neuroendocrine-like. For another six patients, full section immunohistochemistry did not reveal any subtype intra-tumor heterogeneity in the primary tumors (Fig. 4, all cases shown in Supplementary Figure 3).

\section{Lymph-node metastases of Mesenchymal-like and Small-cell/Neuroendocrine-like bladder tumors}

The Mesenchymal-like and Small-cell/Neuroendocrine-like molecular subtypes of bladder cancer comprise only about $5 \%$ each of muscle-invasive bladder tumors. We identified six primary tumors with Mesenchymal-like or Small-cell/ neuroendocrine-like phenotype of which two showed intratumor heterogeneity (Fig. 1a, Supplementary Figure 2). Whenever these minor subtypes were identified in a primary tumor, the lymph-node metastasis also contained tumor of the same subtype. Four of these six cases were identified by pathological examination as histologic variants. Of these, three cases displayed variant histologies both in the primary tumor and the lymph-node metastasis (Supplementary Table 2). One case displayed sarcomatoid variant in the primary tumor only, but this discrepancy could be explained 

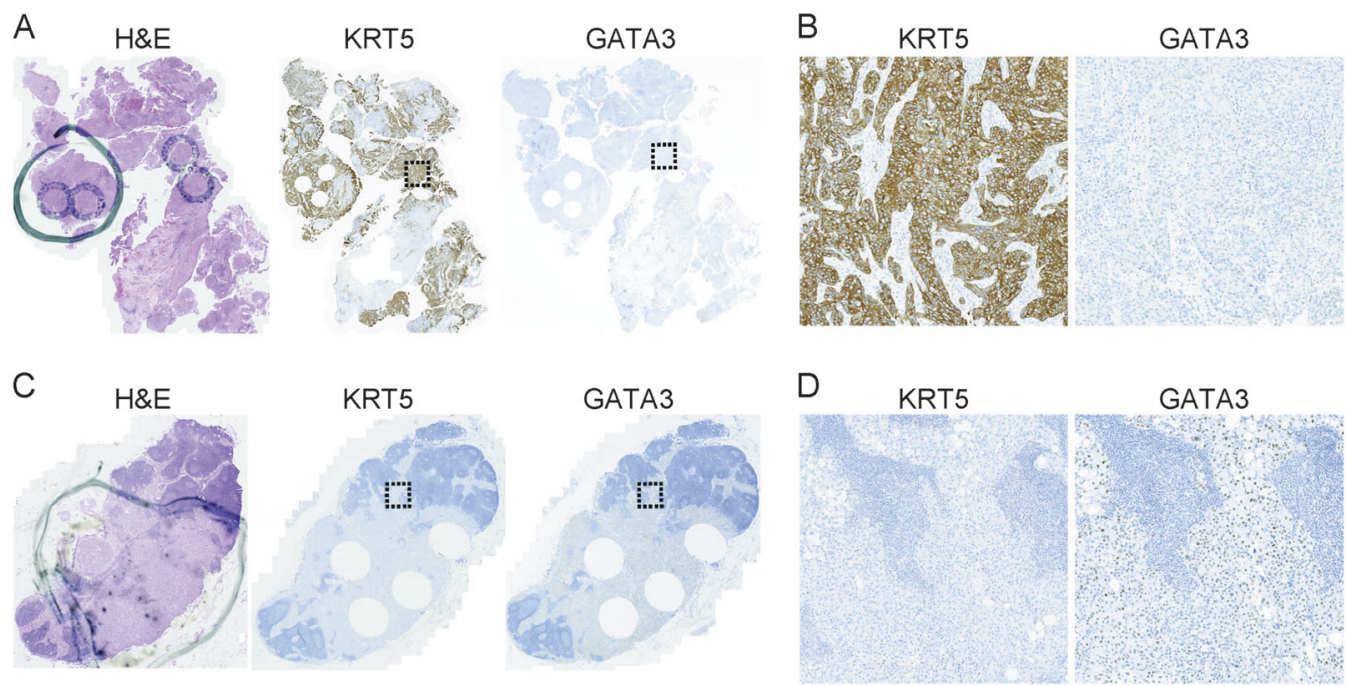

Fig. 4 Full section immunostainings reveal subtype discordance without substantial intra-tumor heterogeneity. a Full section (transurethral resection) stains of a Basal/Squamous-like primary tumor and the discordant Urothelial-like lymph-node metastasis. This case did not show molecular subtype heterogeneity in the primary tumor or in the lymph-node metastasis. On the H\&E stain, the marked areas indicate placement of TMA cores, and macro-dissected areas for DNA/ RNA extraction. b One millimeter squares from the indicated area in the corresponding full sections shown in higher magnification. c No heterogeneity was observed in full section stains of the lymph-node metastasis sample. On the H\&E stain, the marked areas indicate placement of TMA cores, and macro-dissected areas for DNA/RNA extraction. d One millimeter squares from the indicated area in the corresponding full sections shown in higher magnification. Presence of nuclear GATA3 and lack of KRT5 staining confirmed the discordant Urothelial-like subtype classification compared to the Basal/Squamous-like primary tumor. $\mathrm{H} \& \mathrm{E}$ hematoxylin and eosin

markers showed differential expression between primary tumor and metastases (Fig. 5b) and four remained significant after Bonferroni-adjustment, ERBB3, CDH1, and $\mathrm{CDH} 3$ downregulated, and RXRA upregulated in metastases. Except for RXRA, these markers demonstrated high correlation to the corresponding gene in expression data (Pearson- $r$, 0.65-0.79) [28]. Differential protein expression should therefore be reflected as differential mRNA expression. We thus performed similar pairwise $t$-tests in the mRNA data $(n=28)$ for the 10 markers with differential expression. ERBB3,TP63, and ERBB2 mRNA expression were downregulated in metastases also in the mRNA data (Supplementary Figure 4). RXRA, $P P A R G$, and VIM showed opposite pattern in mRNA data compared to immunohistochemistry, indicating a possible differential influence of cells of the different microenvironments on gene expression data. When analyzing the mRNA data set $(n=28$ sample pairs) for significant differential expression, we identified 245 up-, and 74 downregulated genes in lymph-node metastases (paired $t$-test, false discovery rate $<0.01$, Supplementary Table 4) (Fig. 6a). Almost all genes upregulated in metastases, e.g. top genes CCL19, CCL21,CD52, and CXCR4 are expressed by lymphocytes and involved in different aspects of lymph-node function. The genes upregulated in primary tumors demonstrated no obvious biological theme or gene ontology enrichment, but included smooth-muscle (ACTG2) and stromal components 
A

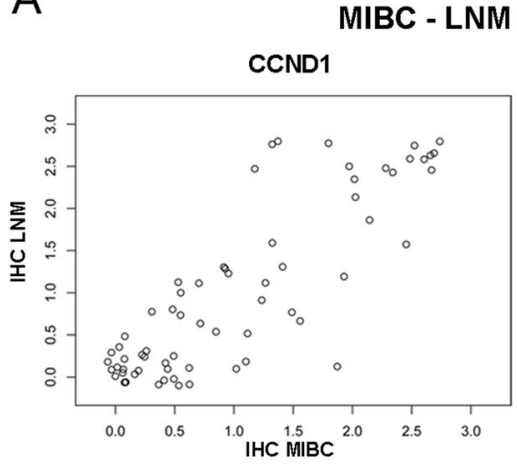

ERBB2

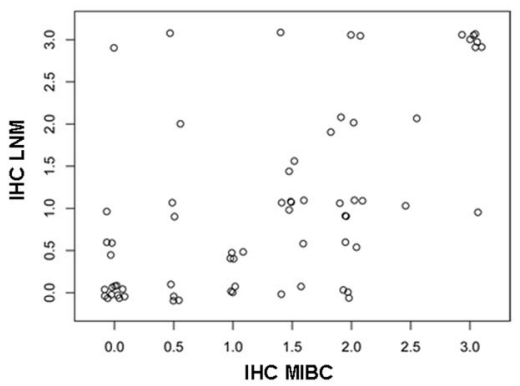

KRT5

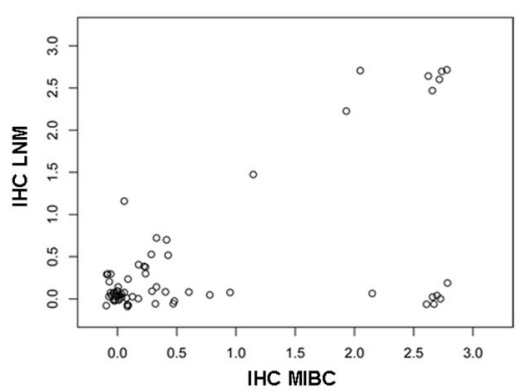

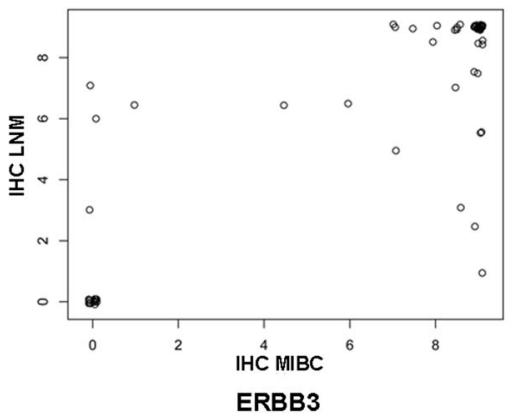

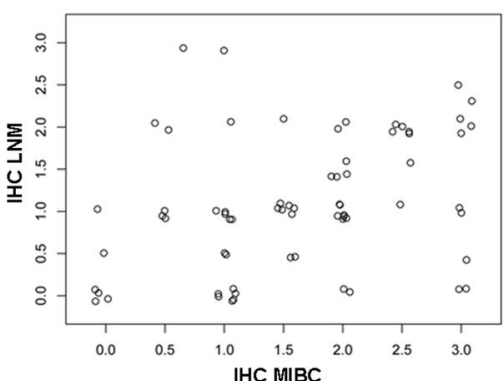

TP63

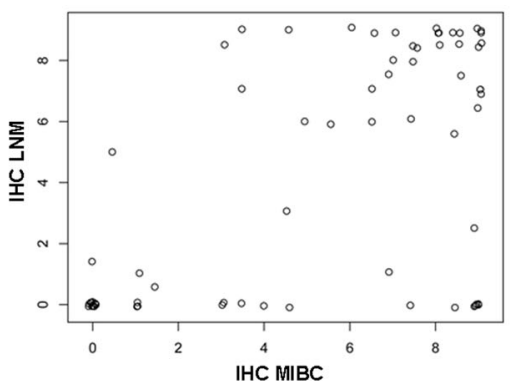

B
Paired t-test (IHC)

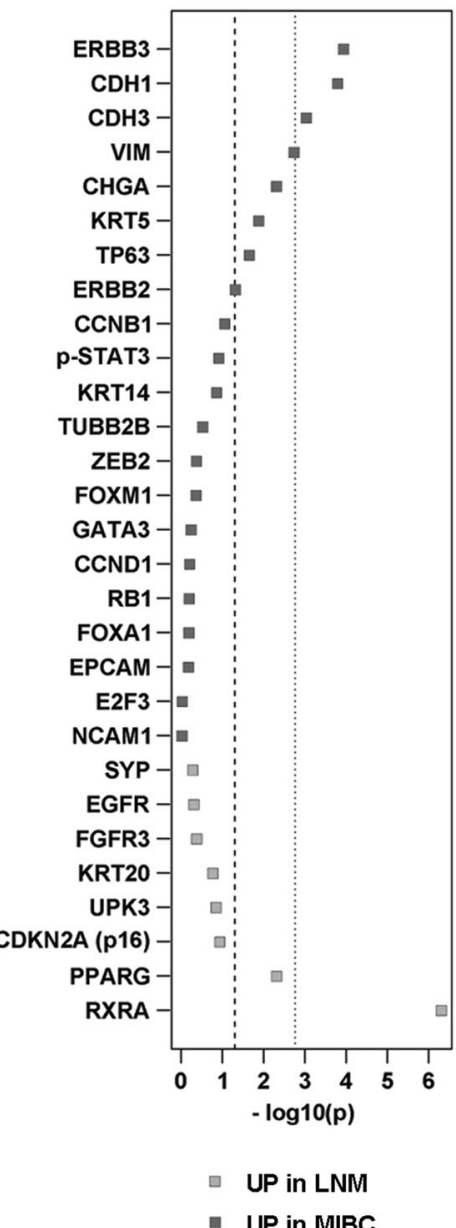

Fig. 5 Differentially expressed proteins in lymph-node metastases versus muscle-invasive bladder tumors. a Different patterns of immunohistochemistry marker expression in primary tumors and lymph-node metastases. CCND1 and RB1 show the strongest correlation between primary tumors and metastases. ERBB2 and ERBB3 show slightly reduced immunostaining in many metastases compared to primary tumors. Basal-markers KRT5 and TP63 show

(LAMA2, COL4A5) expressed in the bladder but absent in pelvic lymph nodes. To increase the chance of detecting genes differentially expressed in the cancer cells, we ordered all significant genes according to their correlation to a cancer immune signature [34] in the extended data set (Fig. 6b). This exploratory analysis identified 58 differentially expressed genes not correlated with the immune signature (absolute $r<0.2$, genes shown as heatmap in Fig. 6c). Although it is not possible to determine which cell type that is responsible for their differential expression, we noted that several of the genes upregulated in lymphnode metastases, e.g. PIK3IP1, TERF $2 I P$, FOXP1, and $M E X 3 C$, have a described negative immune regulatory role in cancer [35-38]. a drop in expression in some metastases corresponding to discordant Basal/Squamous-like cases. b $P$-values from pairwise $t$-test of 29 immunohistochemistry markers between primary tumors and lymph-node metastases. Dotted lines indicate uncorrected and Bonferroni corrected alpha. MIBC muscle-invasive bladder cancer, LNM lymph-node metastasis

\section{Discussion}

Out of 49 cases with a tumor of the Urothelial-like or Genomically Unstable subtypes, only three indicated a phenotypic difference in the lymph-node metastases; one case as a result of intra-tumor subtype heterogeneity of the primary tumor, a second as a result of an atypical phenotype, and a third classified as Urothelial-like in the primary tumor and Basal/Squamous in the lymph-node metastasis. Gene expression profiling reinforced these results, discrepancies compared to immunohistochemistry classification involved mainly the immune-infiltrated subtype and Urothelial-like cases subclassified by global mRNA expression as Urothelial-like $B$. For all six 
A

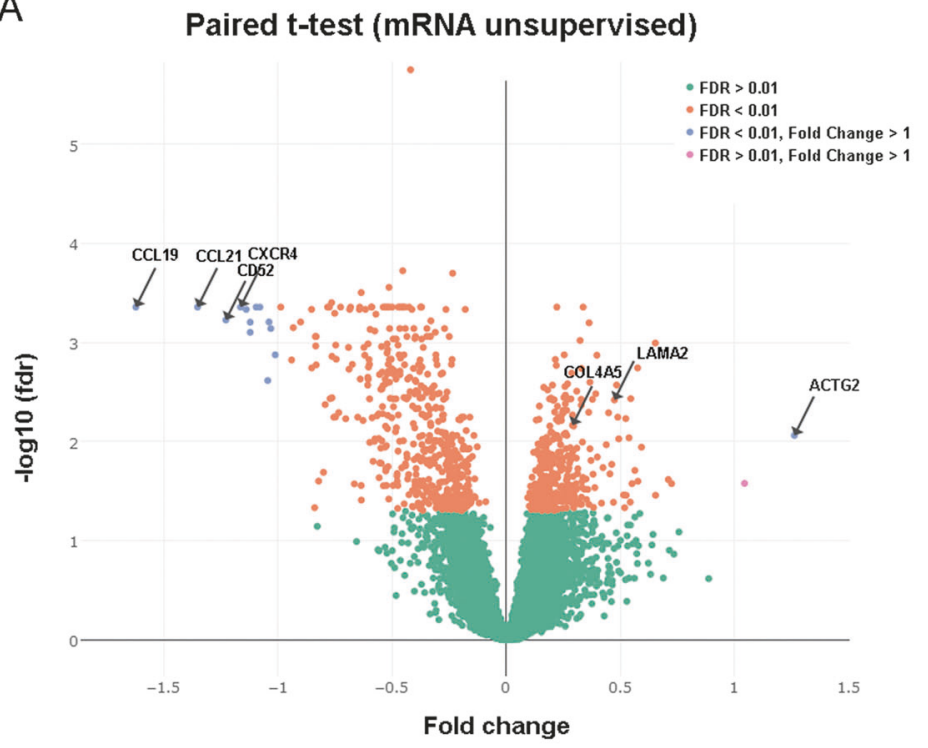

B

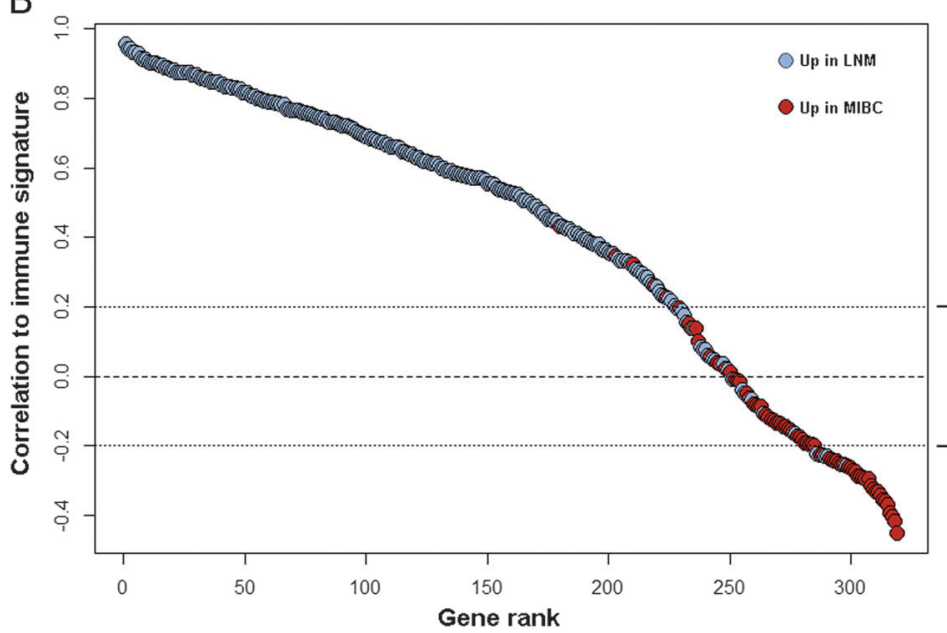

C

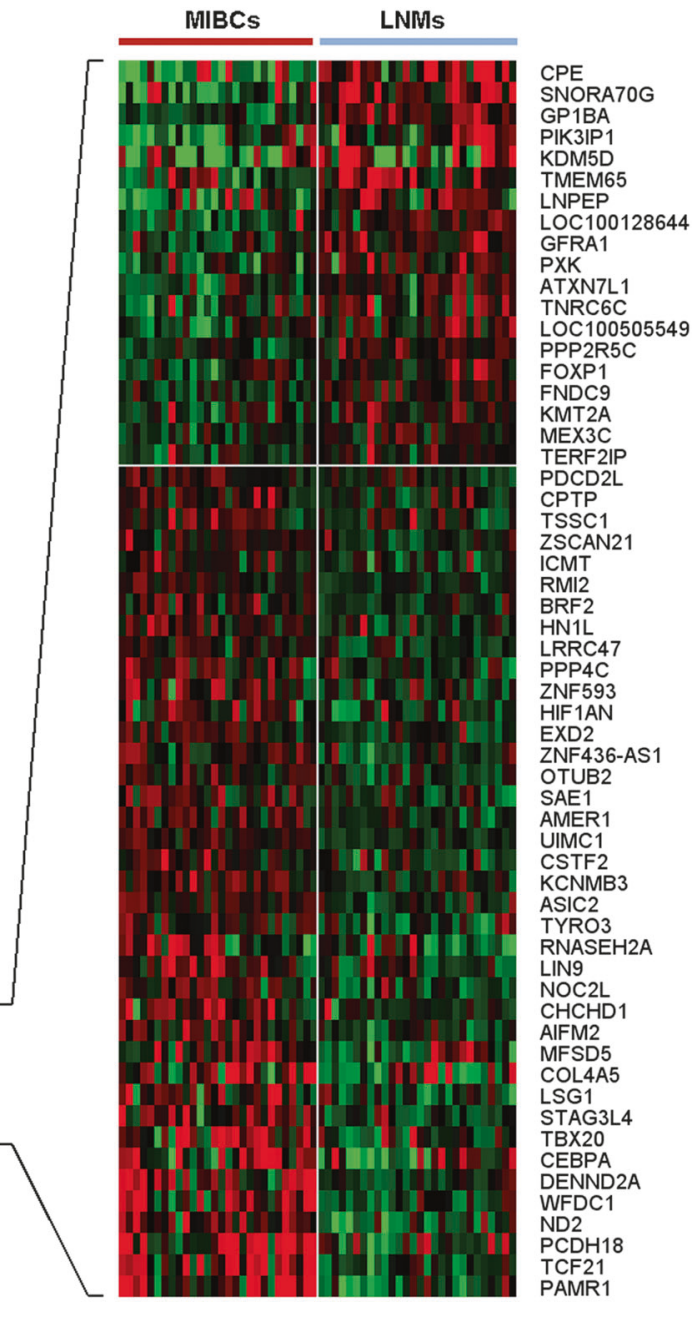

Fig. 6 Differentially expressed genes in lymph-node metastases versus muscle-invasive bladder tumors. a Volcano plot of differential gene expression between primary tumors (positive fold change) and lymphnode metastases (negative fold change). Green dots are non-significant genes, red dots are genes significant at FDR $<0.01$, and gray dots show absolute fold change $>1$. b Significant $(F D R<0.01)$ genes were rank ordered according to their correlation to an immune signature in the extended data set. Genes between the dotted lines were not considered to be affected by immune-cell expression. c Differentially expressed genes, not affected by immune-cell expression, are shown as a heatmap. Primary tumors and metastases are arranged in the same patient order, and genes are ordered by mean fold change between groups. MIBC muscle-invasive bladder cancer, LNM lymph-node metastasis
Mesenchymal-like or Small-cell/neuroendocrine-like cases, the same phenotypes were observed in both samples, but for two of them, the primary tumors showed intra-tumor heterogeneity with a bi-phenotypic appearance. Although the rarity of these subtypes makes the findings anecdotal in nature, we observed Mesenchymal-like and Small-cell/neuroendocrine-like cases with discordant subtype and intratumor heterogeneity, but also fully concordant cases, e.g. a Small-cell/neuroendocrine-like case with shared inactivating mutations in both RBI and NCOR1. Tumors of the Basal/Squamous-like subtype were the only group that showed substantial subtype discordance in lymph-node metastases. More than half (7 of 12) of the Basal/Squamous-like cases were classified as Urothelial-like or Genomically Unstable in the matched metastasis samples, but full section evaluations revealed subtype intra-tumor heterogeneity to underlie/explain four of these shifts. As even full sections represent only a small sample of a tumor, we cannot exclude that more, discordant cases could be explained by subtype intra-tumor heterogeneity. The opposite is also possible, i.e. that tumor cells of discordant cases have changed subtype, possibility by interactions with microenvironments in the two anatomical sites. The latter concept has some support in experimental data from breast 
cancer where basal- and luminal-like cell lines may change to the opposite subtype when forming tumors in the mammary duct, or in the mammary fat pad, respectively [39]. Further support for the dynamic nature of the basallike phenotype in cancer was provided by Cheung et al. [40] who showed that luminal-like breast cancer cells can undergo phenotypic conversion to basal-like cells during collective invasion, and that this process depended on the matrix composition. Here, we could show that Basal/Squamous-like bladder tumors were consistently classified by mRNA and immunohistochemistry, validating subtype classification for three concordant and one discordant case. A larger, confirmatory analysis of tumors and metastases, focusing on Basal/Squamous-like tumors, seems to be required since the current study was not selected to specifically include tumors of this subtype. Preferably, such a study would include distant as well as regional metastases to test whether this finding can be validated in both settings.

Our results also point towards a possible overestimation of subtype shifts when determined by global mRNA classification of paired tumor samples. Direct application of a centroid classifier (Supplementary Figure 1) without quantification or validation by another method resulted in almost as many false subtype shifts due to threshold effects, as true subtype shifts. This finding underscores the importance of applying several classification methods, validating mRNA classification by immunohistochemistry, and using quantitative assessment of subtype classification, particularly when comparing paired samples. This observation most likely holds true for primary tumor/metastasis comparisons, as exemplified here, as well as for comparisons before and after treatment.

Most high confidence mutations were shared between sample pairs, suggesting that lymph-node metastases may not diverge as early from bladder tumors as has been suggested for hematogenous metastases [41, 42]. Nearly half $(8 / 20)$ of analyzed cases had mutations in KDM6A, which is frequently mutated in the most ancestral bladder cancer clone [43]. Altogether, the data are consistent with lymphnode metastases being genetically similar to the main clone, or bulk of bladder tumor cells, as opposed to a small subclonal population of metastasis-generating cells. Particular insight was gained from the Basal/Squamous-like case shifting to Genomically Unstable phenotype on both immunohistochemistry and mRNA classification. The driver mutation V104L in ERBB3 was detected in both phenotypes and in the two anatomic sites, thus favoring a genuine phenotypic shift over parallel evolution.

Only three genes, ERBB2, ERBB3, and TP63, were differentially expressed at both protein and mRNA levels in the primary tumor and metastasis. The lower expression of ERBB2 in lymph-node metastases is in conflict with some $[16,17]$ but not all [18] previous data. By unsupervised gene expression comparisons we identified differentially regulated genes. While these lists may contain genes functionally involved in the establishment and maintenance of metastases, they were definitely also enriched for (immune) cell-type associated genes. After exploratory removal of the vast majority of genes with immune-cell associated expression, several genes, e.g. PIK3IP1, TERF2IP, FOXP1, and $M E X 3 C$, with potentially immune regulatory functions remained [35-38].

Even though the current study is performed in a population-based cohort, it is limited by selection mechanisms related to tissue availability for the different molecular analyses used. Furthermore, possible influence on selection mechanisms and clonal evolution due to perioperative chemotherapy in eight cases cannot be ruled out [44].

An urgent goal for clinical bladder cancer research is to predict response to systemic chemotherapy and immunecheckpoint inhibitors. Early indications show that molecular subtypes could play a role in response prediction for both treatment modalities $[3,9,10]$. So far, such studies have used samples from the primary tumor, assuming that these are representative for the residual disease that causes postcystectomy recurrence. Although our data generally support this assumption, we also question the intrinsic nature of the Basal/Squamous-like subtype. Studies investigating the Basal/Squamous-like subtype and its associated drug targets (e.g. EGFR, MET, STAT3) should take bladder tumor heterogeneity as well as discordance of molecular subtype in metastases into consideration. Furthermore, our data may suggest that the discordance of Basal/Squamous-like samples could depend on interaction with the microenvironment in different anatomic sites. Identification of such governing factor(s) may then improve our understanding of the Basal/ Squamous phenotype in bladder cancer.

Acknowledgements Microarray data were generated by the SCIBLU genomics facility in Lund, Sweden. This research was supported by grants from the Swedish Cancer Society (2016/314 and 2017/278), Lund Medical Faculty (ALF) (2014/354), Skåne University Hospital Research Funds, Sten K Johnson Foundation, The Krapperup Foundation, Skane County Council's research and development Foundation REGSKANE-622351, Gösta Jönsson Research Foundation, and Biocare. The authors wish to thank Jaegil Kim for performing TCGA subtype classification.

\section{Compliance with ethical standards}

Conflict of interest The authors declare that they have no conflict of interest.

\section{References}

1. Madersbacher S, Hochreiter W, Burkhard F, et al. Radical cystectomy for bladder cancer today-a homogeneous series without neoadjuvant therapy. J Clin Oncol. 2003;21: 690-6. 
2. Grossman HB, Natale RB, Tangen CM, et al. Neoadjuvant chemotherapy plus cystectomy compared with cystectomy alone for locally advanced bladder cancer. N Engl J Med. 2003;349: 859-66.

3. Rosenberg JE, Hoffman-Censits J, Powles T, et al. Atezolizumab in patients with locally advanced and metastatic urothelial carcinoma who have progressed following treatment with platinumbased chemotherapy: a single-arm, multicentre, phase 2 trial. Lancet. 2016;387:1909-20.

4. Bellmunt J, de Wit R, Vaughn DJ, et al. Pembrolizumab as second-iine therapy for advanced urothelial carcinoma. N Engl $\mathbf{J}$ Med. 2017;376:1015-26.

5. Balar AV, Galsky MD, Rosenberg JE, et al. Atezolizumab as firstline treatment in cisplatin-ineligible patients with locally advanced and metastatic urothelial carcinoma: a single-arm, multicentre, phase 2 trial. Lancet. 2017;389:67-76.

6. International Collaboration of Trialists, Griffiths G, Hall R, Sylvester $\mathrm{R}$, et al. International phase III trial assessing neoadjuvant cisplatin, methotrexate, and vinblastine chemotherapy for muscleinvasive bladder cancer: long-term results of the BA06 30894 trial. J Clin Oncol. 2011;29:2171-7.

7. Font A, Taron M, Gago JL, et al. BRCA1 mRNA expression and outcome to neoadjuvant cisplatin-based chemotherapy in bladder cancer. Ann Oncol. 2011;22:139-44.

8. Baras AS, Gandhi N, Munari E, et al. Identification and validation of protein biomarkers of response to neoadjuvant platinum chemotherapy in muscle invasive urothelial carcinoma. PLoS ONE. 2015;10:e0131245.

9. Choi W, Porten S, Kim S, et al. Identification of distinct basal and luminal subtypes of muscle-invasive bladder cancer with different sensitivities to frontline chemotherapy. Cancer Cell. 2014;25: 152-65.

10. Seiler R, Ashab HA, Erho N, et al. Impact of molecular subtypes in muscle-invasive bladder cancer on predicting response and survival after neoadjuvant chemotherapy. Eur Urol. 2017;72: 544-54.

11. Van Allen EM, Mouw KW, Kim P, et al. Somatic ERCC2 mutations correlate with cisplatin sensitivity in muscleinvasive urothelial carcinoma. Cancer Discov. 2014;4:1140-53.

12. Plimack ER, Dunbrack RL, Brennan TA, et al. Defects in DNA repair genes predict response to neoadjuvant cisplatin-based chemotherapy in muscle-invasive bladder cancer. Eur Urol. 2015;68: 959-67.

13. Groenendijk FH, de Jong J,Fransen van de Putte EE, et al. ERBB2 mutations characterize a subgroup of muscle-invasive bladder cancers with excellent response to neoadjuvant chemotherapy. Eur Urol. 2016;69:384-8.

14. Badalament RA, O'Toole RV, Keyhani-Rofagha S, et al. Flow cytometric analysis of primary and metastatic bladder cancer. J Urol. 1990;143:912-5.

15. Jones TD, Carr MD, Eble JN, et al. Clonal origin of lymph node metastases in bladder carcinoma. Cancer. 2005;104:1901-10.

16. Jimenez RE, Hussain M, Bianco FJ Jr, et al. Her-2/neu over-expression in muscle-invasive urothelial carcinoma of the bladder: prognostic significance and comparative analysis in primary and metastatic tumors. Clin Cancer Res. 2001;7: 2440-7.

17. Fleischmann A, Rotzer D, Seiler R, et al. Her2 amplification is significantly more frequent in lymph node metastases from urothelial bladder cancer than in the primary tumours. Eur Urol. 2011;60:350-7.

18. Hansel DE, Swain E, Dreicer R, et al. HER2 overexpression and amplification in urothelial carcinoma of the bladder is associated with MYC coamplification in a subset of cases. Am J Clin Pathol. 2008;130:274-81.
19. Carlsson J, Wester K, De La Torre M, et al. EGFR-expression in primary urinary bladder cancer and corresponding metastases and the relation to HER2-expression. On the possibility to target these receptors with radionuclides. Radiol Oncol. 2015;49: $50-8$.

20. Turo R, Harnden P, Thygesen $\mathrm{H}$, et al. FGFR3 expression in primary invasive bladder cancers and matched lymph node metastases. J Urol. 2015;193:325-30.

21. Pouessel D, Neuzillet Y, Mertens LS, et al. Tumor heterogeneity of fibroblast growth factor receptor 3 (FGFR3) mutations in invasive bladder cancer: implications for perioperative antiFGFR3 treatment. Ann Oncol. 2016;27:1311-6.

22. Byrne RR, Shariat SF, Brown R, et al. E-cadherin immunostaining of bladder transitional cell carcinoma, carcinoma in situ and lymph node metastases with long-term followup. J Urol. 2001; 165:1473-9.

23. Seiler R, Thalmann GN, Rotzer D, et al. CCND1/ CyclinD1 status in metastasizing bladder cancer: a prognosticator and predictor of chemotherapeutic response. Mod Pathol. 2014; 27:87-95.

24. Damrauer JS, Hoadley KA, Chism DD, et al. Intrinsic subtypes of high-grade bladder cancer reflect the hallmarks of breast cancer biology. Proc Natl Acad Sci USA. 2014;111:3110-5.

25. Sjödahl G, Lauss M, Lövgren K, et al. A molecular taxonomy for urothelial carcinoma. Clin Cancer Res. 2012;18:3377-86.

26. Robertson AG, Kim J, Al-Ahmadie H, et al. Comprehensive molecular characterization of muscle-invasive bladder cancer. Cell. 2017;171:540-56.

27. Dadhania V, Zhang M, Zhang L, et al. Meta-analysis of the luminal and basal subtypes of bladder cancer and the identification of signature immunohistochemical markers for clinical use. EBioMedicine. 2016;12:105-17.

28. Sjödahl G, Eriksson P, Liedberg F, et al. Molecular classification of urothelial carcinoma: global mRNA classification versus tumour-cell phenotype classification. J Pathol. 2017;242:113-25.

29. Lee JY, Park K, Lee E, et al. Gene expression profiling of breast cancer brain metastasis. Sci Rep. 2016;6:28623.

30. Priedigkeit N, Hartmaier RJ, Chen Y, et al. Intrinsic subtype switching and acquired ERBB2/HER2 amplifications and mutations in breast cancer brain metastases. JAMA Oncol. 2017;3: 666-71.

31. Forbes SA, Beare D, Boutselakis H, et al. COSMIC: somatic cancer genetics at high-resolution. Nucleic Acids Res. 2017;45: D777-83.

32. Lek M, Karczewski KJ, Minikel EV, et al. Exome Aggregation Consortium. Analysis of protein-coding genetic variation in 60,706 humans. Nature. 2016;536:285-91.

33. Adzhubei IA, Schmidt S, Peshkin L, et al. A method and server for predicting damaging missense mutations. Nat Methods. 2010;7:248-9.

34. Yoshihara K, Shahmoradgoli M, Martínez E, et al. Inferring tumour purity and stromal and immune cell admixture from expression data. Nat Commun. 2013;4:2612.

35. DeFrances MC, Debelius DR, Cheng J, et al. Inhibition of T-cell activation by PIK3IP1. Eur J Immunol. 2012;42:2754-9.

36. Teo H, Ghosh S, Luesch H, et al. Telomere-independent Rap1 is an IKK adaptor and regulates NF-kappaB-dependent gene expression. Nat Cell Biol. 2010;12:758-67.

37. Stephen TL, Rutkowski MR, Allegrezza MJ, et al. Transforming growth factor $\beta$-mediated suppression of antitumor T cells requires FoxP1 transcription factor expression. Immunity. 2014;41: 427-39.

38. Cano F, Bye H, Duncan LM, et al. The RNA-binding E3 ubiquitin ligase MEX-3C links ubiquitination with MHC-I mRNA degradation. EMBO J. 2012;31:3596-606. 
39. Sflomos G, Dormoy V, Metsalu T, et al. A preclinical model for ER $\alpha$-positive breast cancer points to the epithelial microenvironment as determinant of luminal phenotype and hormone response. Cancer Cell. 2016;29:407-22.

40. Cheung KJ, Gabrielson E, Werb Z, et al. Collective invasion in breast cancer requires a conserved basal epithelial program. Cell. 2013;155:1639-51.

41. Thomsen MB, Nordentoft I, Lamy P, et al. Spatial and temporal clonal evolution during development of metastatic urothelial carcinoma. Mol Oncol. 2016;10:1450-60.
42. Faltas BM, Prandi D, Tagawa ST, et al. Clonal evolution of chemotherapy-resistant urothelial carcinoma. Nat Genet. 2016;48:1490-9.

43. Nordentoft I, Lamy P, Birkenkamp-Demtröder K, et al. Mutational context and diverse clonal development in early and late bladder cancer. Cell Rep. 2014;7:1649-63.

44. Liu D, Abbosh P, Keliher D, et al. Mutational patterns in chemotherapy resistant muscle-invasive bladder cancer. Nat Commun. 2017;8:2193. 